\title{
Overcoming the Challenges of Beam-sensitivity in Fuel Cell Electrodes
}

\author{
David A. Cullen ${ }^{1}$, Brian T. Sneed ${ }^{2}$, and Karren L. More ${ }^{2}$ \\ 1. Materials Science \& Technology Division, ORNL, Oak Ridge, TN USA \\ 2. Center for Nanophase Materials Sciences, ORNL, Oak Ridge, TN USA
}

The materials comprising polymer electrolyte membrane fuel cells (PEMFCs) offer a host of imaging challenges, spanning wide length scales and materials classes. Issues of beam sensitivity are greatly magnified when scanning transmission electron microscopy (STEM)-based spectroscopic techniques, such as electron energy loss spectroscopy (EELS) or energy dispersive X-ray spectroscopy (EDS), are employed. This work will present our investigations in mitigating beam artefacts in the study of PEMFC catalyst layers.

Thin perfluorosulfonic acid (PFSA) ionomer layers serve the critical role of proton conduction within the fuel cell electrodes, but these films are very sensitive to radiolysis during examination under the electron beam [1]. Previously employed strategies for reducing beam damage in such fluorinated compounds include cryogenic cooling, sputter coating electrically conductive surface layers, increasing the accelerating voltage, and limiting the electron dose [2,3]. The different strategies were investigated for the case of thin PFSA ionomer films. The dose required for EELS and EDS spectrum imaging was quantitatively compared, and multivariate statistical analysis was employed to improve signal-to-noise and to further reduce electron dose, as shown in Figure 1.

Although typically stable under standard STEM imaging conditions, precious metal catalytic nanoparticles can also suffer from seldom-reported beam damage artefacts under the high doses required for either longer-term electron tomography or EDS mapping experiments, with the effect increasing with decreasing particle sizes. As shown in Figure 2, both supported and non-supported catalyst particles readily suffer from beam damage, which cannot be mitigated by simply changing the accelerating voltage. In the case of fuel cell catalysts, alloy nanoparticles are often utilized, in which the compositional variations on the $\AA$-scale can have a significant impact on the catalyst activity. The identification and mitigation of artefacts arising from electron beam induced damage in 2D spectrum images and 3D tomographic reconstructions in nanoparticle alloy catalysts will be presented.

Finally, the case of single-atom catalysis will be discussed. The active sites in platinum group metal free (PGM-free) fuel cell catalysts are believed to be individual Fe atoms coordinated by $\mathrm{N}$ [4], e.g., $\mathrm{FeN}_{\mathrm{x}}$ complexes, bound by carbon. While Z-contrast STEM imaging of individual metallic atoms on single-layer carbon supports is relatively straightforward, probing the bonding environment of such atoms is extremely challenging due to their instability and movement under the electron beam. Similar to the graphene-stabilizing effect previously observed for $\mathrm{MoS}_{2}$, the encapsulation of Fe within or between graphene layers will lead to increased stability under the low voltage, aberration-corrected electron beam [5]. Quantitative EELS spectrum images will be presented that demonstrate how encapsulation within graphene allows for the direct observation of these model-predicted sites.[6]

\section{References:}

[1] DA Cullen et al, J. Electrochem. Soc. 161 (2014), p. F1111. 
[2] BJ Ciliax, KL Kirk, and RD Leapman, Ultramicroscopy 48 (1993), p. 13.

[3] S Yakovlev and KH Downing, Phys. Chem. Chem. Phys. 15 (2013), p. 1052.

[4] EFZ. Holby et al., Nano Energy 29 (2016) p.54

[5] R Zhan et al, ACS Nano 7 (2013), p.10167.

[6] Research sponsored by the Fuel Cell Technologies Office, Office of Energy Efficiency and Renewable Energy, U.S. Department of Energy (DOE) and ORNL's Center for Nanophase Materials Sciences (CNMS), which is a DOE Office of Science User Facility
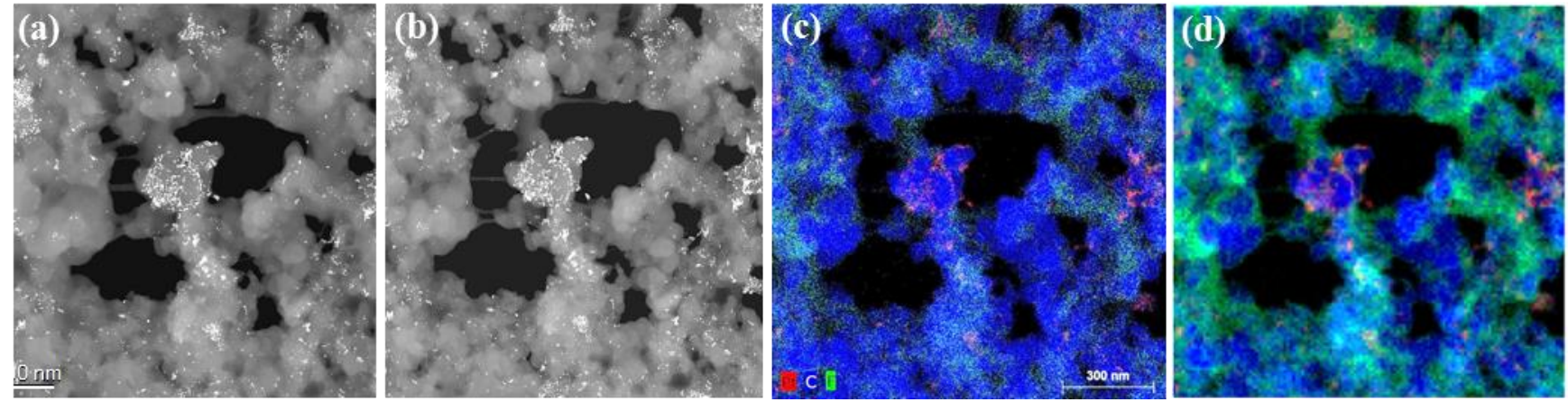

Figure 1. Cryo-STEM EDS spectrum imaging of a porous fuel cell electrode. HAADF-STEM images (a) prior to and (b) after spectrum imaging with a total dose of $1 \times 10^{6} \mathrm{e}^{-} / \mathrm{nm}^{2}$. Overlaid spectrum images of C (blue), Pt (red), and F (green) generated from (c) raw data and (d) principal component analysis [1].
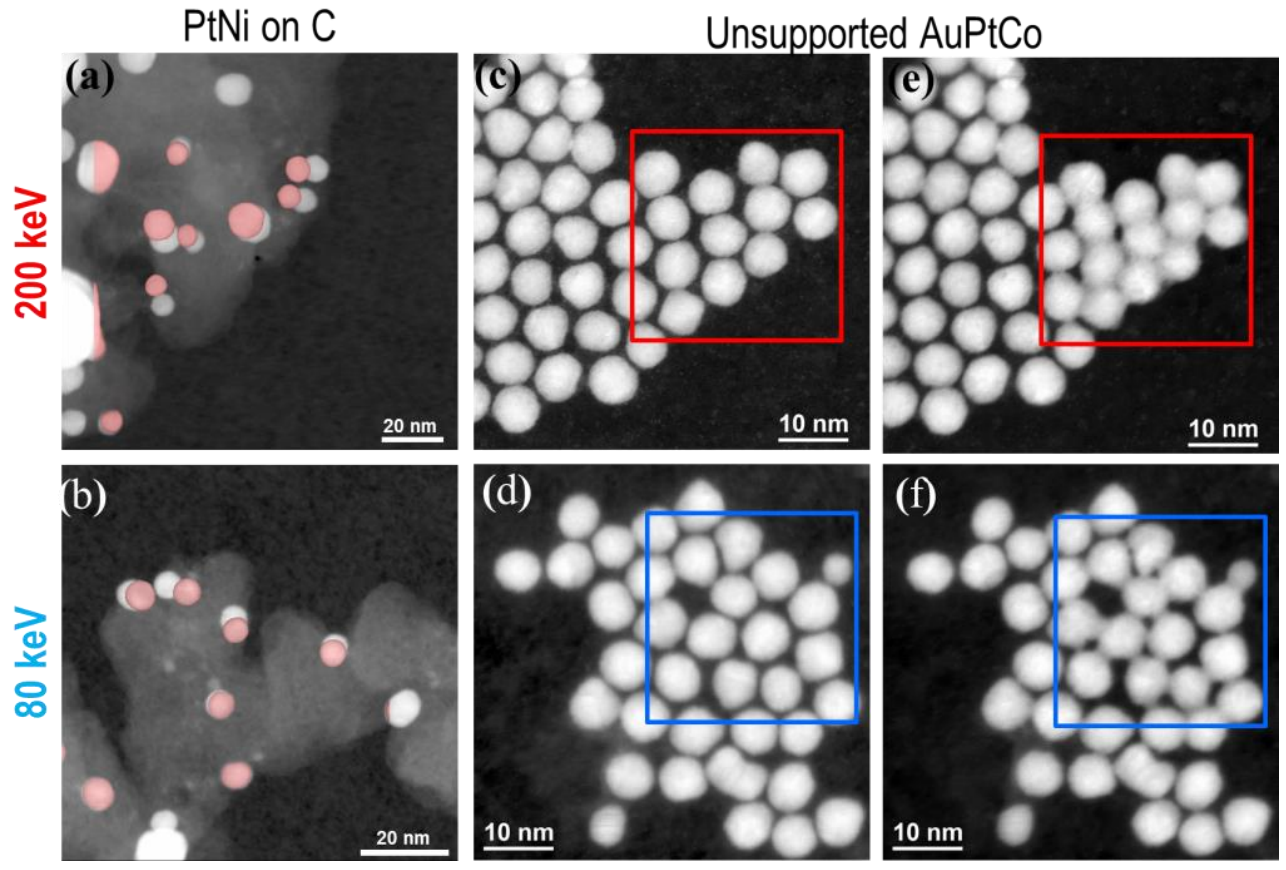

Figure 2. Overlaid HAADF-STEM images of PtNi catalysts imaged before (white) and after (pink) EDS spectrum imaging. Particle movement on the carbon support was observed at both (a) $200 \mathrm{kV}$ and (b) $80 \mathrm{kV}$. (c-f) Sintering of AuPtCo particles during STEM-EDS mapping at both 200kV (c,e) and $80 \mathrm{kV}(\mathrm{d}, \mathrm{f})$. The boxes mark the region used for mapping. 